\title{
Collagen Pores Determined by Electron Microscopy
}

\author{
Max Swerdlow and Robert R. Stromberg
}

\begin{abstract}
The existence and morphology of pores of the order of $150 \mathrm{~A}$, in radius, within collagen fibrils is delineated. Structural features of collagen from air-dried kangaroo tail tendon, impregnated with mercury at a pressure of 10,800 psi., are compared with those of fibrils not exposed to mercury. A helical configuration of subfibrillar elements is suggested by the electron microgaphs of individual fibrils. The possible use of mercury under hydrostatic pressure as a technique in staining and preserving the structure of biological materials for electron microscopy is advanced. These findings constitute a visual confirmation of the presence and probable size of small pores deduced from theoretical considerations of poresize distributions in collagen and leather, and provide additional information about the shape, location, and arrangement of such pores in collagen fibrils.
\end{abstract}

\section{Introduction}

Physical methods of characterization have demonstrated that, in general, native collagen from different animals and from different tissues (skin, tendon, bone, and other connective tissues) have, in common, a characteristic axial periodicity varying from 600 to $675 \mathrm{~A}$. Electron microscopy has provided direct visualization of still finer periodic structures within these periodic bands. Even though a comprehensive literature of collagen exists [1], ${ }^{1}$ additional data and fundamental concepts concerning the morphogensis and basic structure are still being sought [2]. Recently, the structure of collagen has been amply reviewed and evaluated [3, 4, 5, 5a]. X-ray diffraction data have been interpreted to suggest a helical configuration of the molecular polypeptide chains around a central axis $[6,7,8]$. These helices are considered to be discontinuous, and models have been suggested for the structure of parts of the collagen molecule. These models represent a scale at least an order of magnitude smaller than the subfibrillar elements observed in the electron microscope.

A variety of terms have been used in describing the morphological components and the structures observed in the electron microscope and interpreted from X-ray diffraction data. The nomenclature and definitions used by Bear [3] have been generally adopted for this paper. The hypothetical thinnest filamentous unit, the protofibril, first suggested by Schmitt, Hall, and Jakus [9], represents the smallest coherent unit that carries the essential chemical and configurational structure of collagen. It has been suggested that the protofibril is no wider than a very few polypeptide chains (of the order 12 to $17 \mathrm{~A}$ in diameter). In the theoretical development, protofibrils combine to form the wider units observed in the electron microscope. The smallest structures presently resolved by means of electron microscopy have been termed filaments. (The thinnest are about $50 \mathrm{~A}$ in diameter.) The larger fibrous structures observed in the electron microscope have been termed fibrils, each of which is of constant diameter, but varying in size from fibril to fibril (of the order 200

Figures in brackets indicate the literature references at the end of this paper. to $2000 \mathrm{~A}$ in diameter). These fibrils aggregate to form the much larger primitive fibers (2 to $10 \mu$ in diameter). These in turn form fibers with diameters of 100 to $200 \mu$ in tendon and 20 to $40 \mu$ in skin.

The properties of leather are fundamentally related to the properties of collagen, which is the basic constituent of the tissue from which leather is derived. Many of these properties are attributable, to a large extent, to the porous network within the fibrous matrix. For example, the ability of tanning agents or polymers [10] to impregnate raw hide and leather is directly related to this porous. structure. A previous study of the pore-size distributions in collagen and leather by the porosimeter method [11] resulted in two general conclusions that were applicable to all materials investigated: (1) mercury was retained within the capillary structure of the porous material, and (2) a significant volume was concentrated in the small pore-size range, 100 to $1000 \mathrm{~A}$, in radius. It would be desirable to have direct visual evidence of the nature of such pores.

The present electron microscopical investigation is, therefore, concerned primarily with the geometry of the collagen fibril insofar as it relates to the existence of pores within the structure and to the location, size, and arrangement of such pores in collagen. For this purpose, kangaroo tail tendon was selected for study because it is considered to be similar to other mammalian collagen and is available in a relatively pure state. This material, selected from the variety of collagenous tissues for which poresize distributions were obtained [11], ofters additional advantages from the standpoint of its relatively large size and readily discernible structure. Although the data and interpretations obtained from the air-dried material chosen for this investigation may not be valid for collagen in the living state, it is believed that this material more nearly resembles the collagen in leather.

\section{Experimental Procedure}

In the porosimeter method [11], bulk pieces of air-dried kangaroo tail tendon were evacuated to a pressure of about $10^{-5} \mathrm{~mm}$ of $\mathrm{Hg}$, immersed in mercury, and subjected to hydrostatic pressures as high 

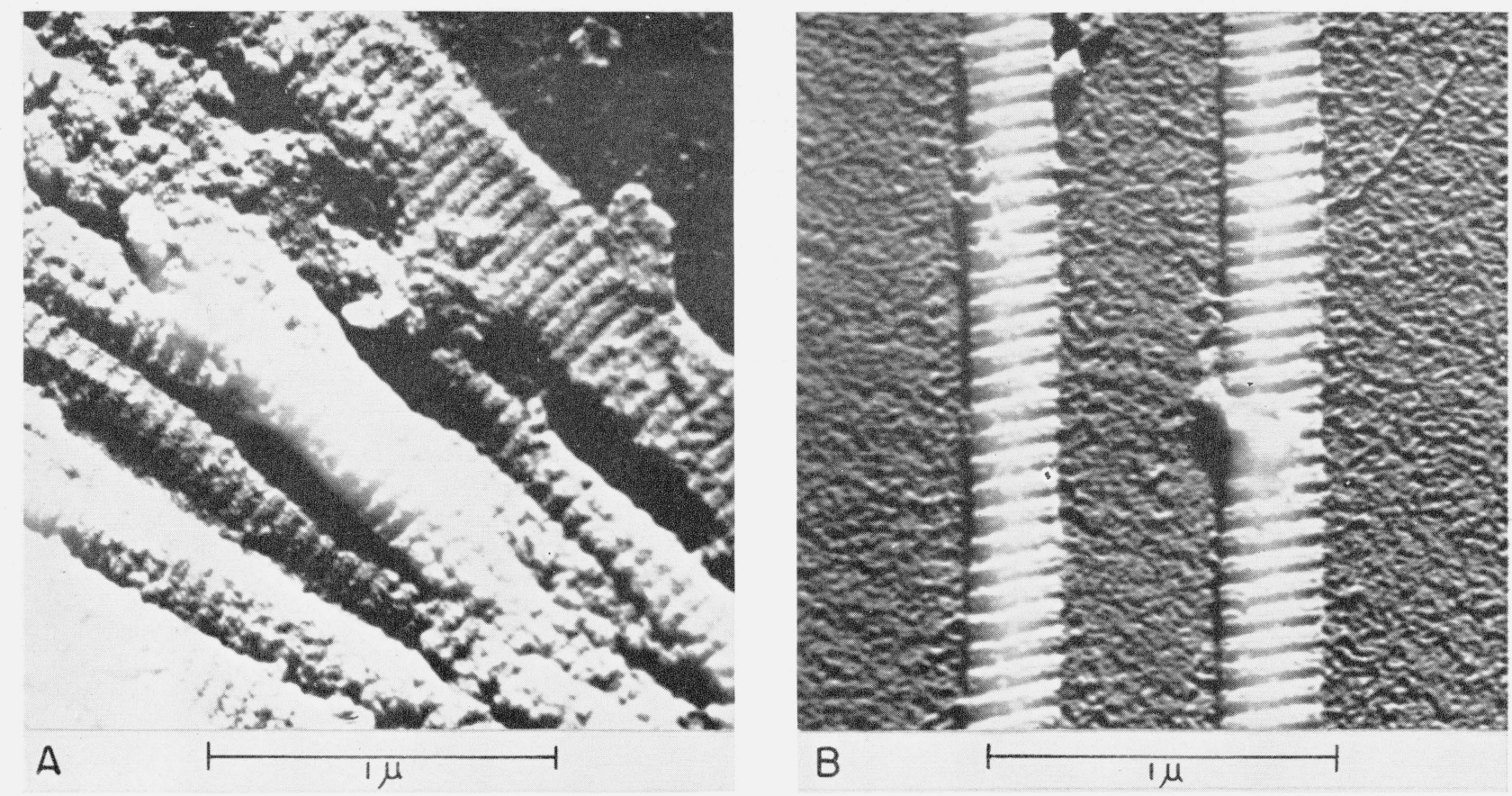

B

$1 \mu$
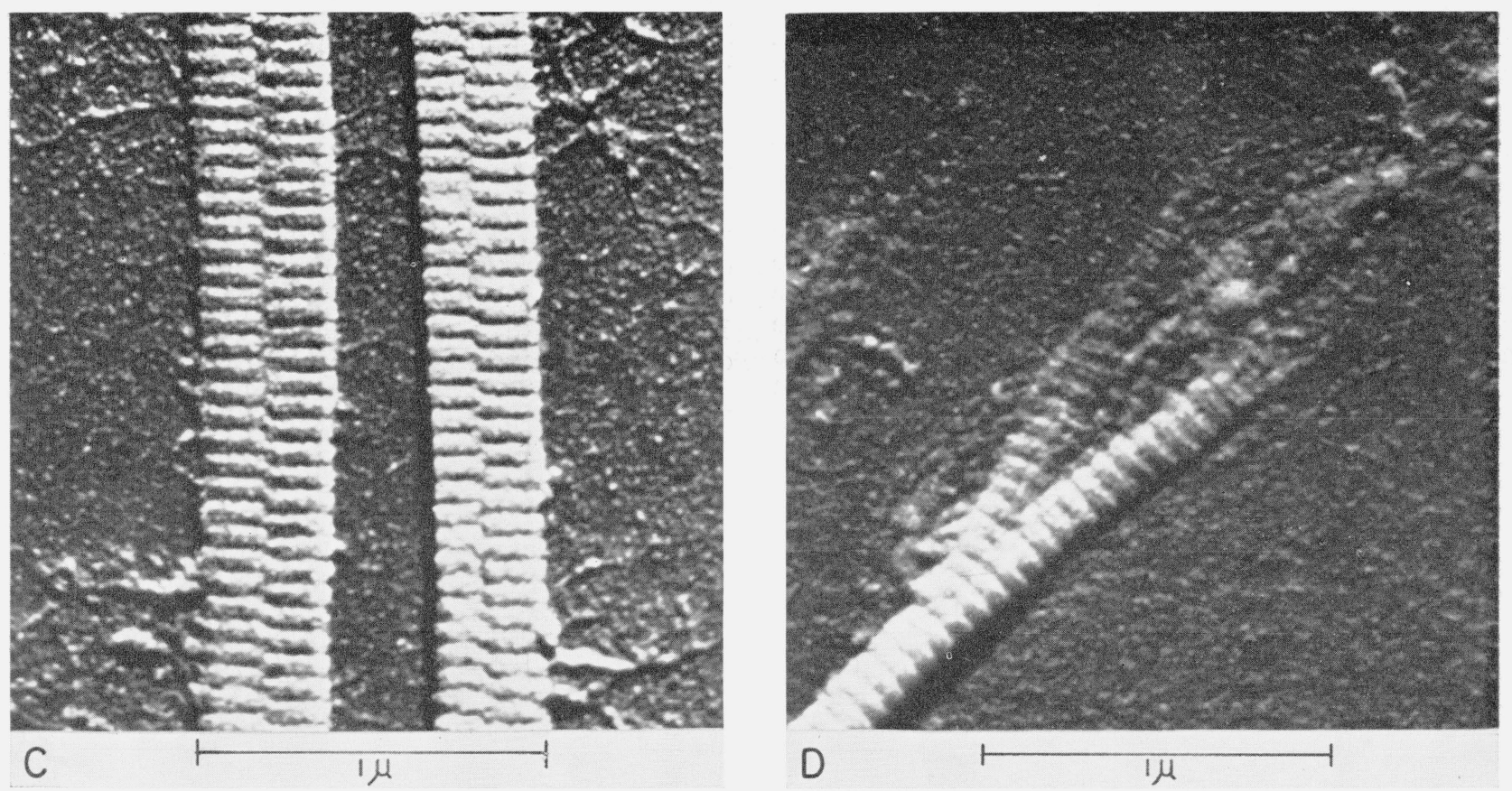

FIGURE 1. Electron micrographs of palladium-shadowed specimens of collagen from kangaroo tail tendon dispersed in water.

A. Poorly dispersed mass of many collagen fibrils.

C. Two pairs of collagen fibrils.
B. Two individual collagen fibrils with characteristic cross striations, D. Frayed ends of one or more collagen fibrils. 

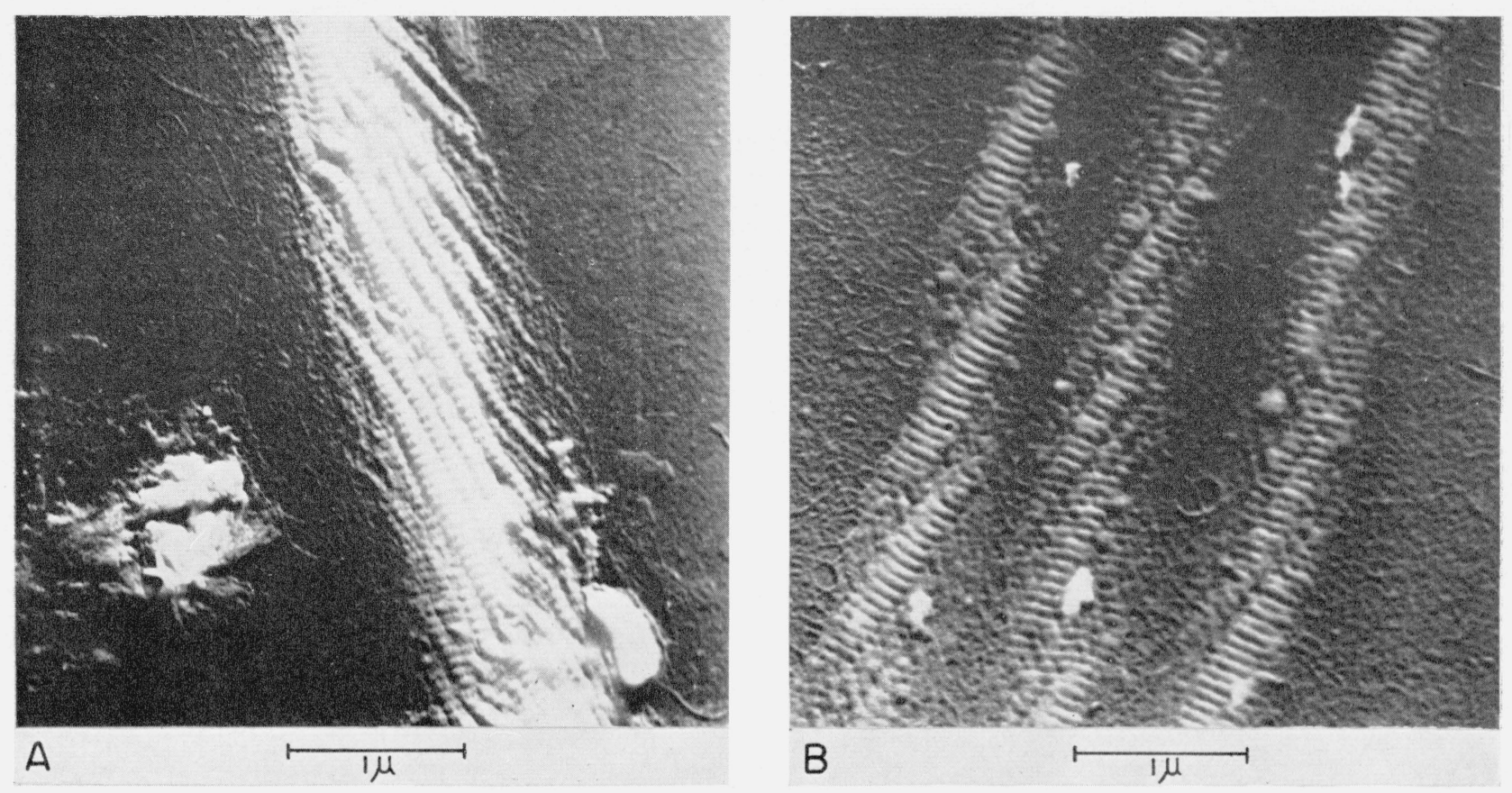

B
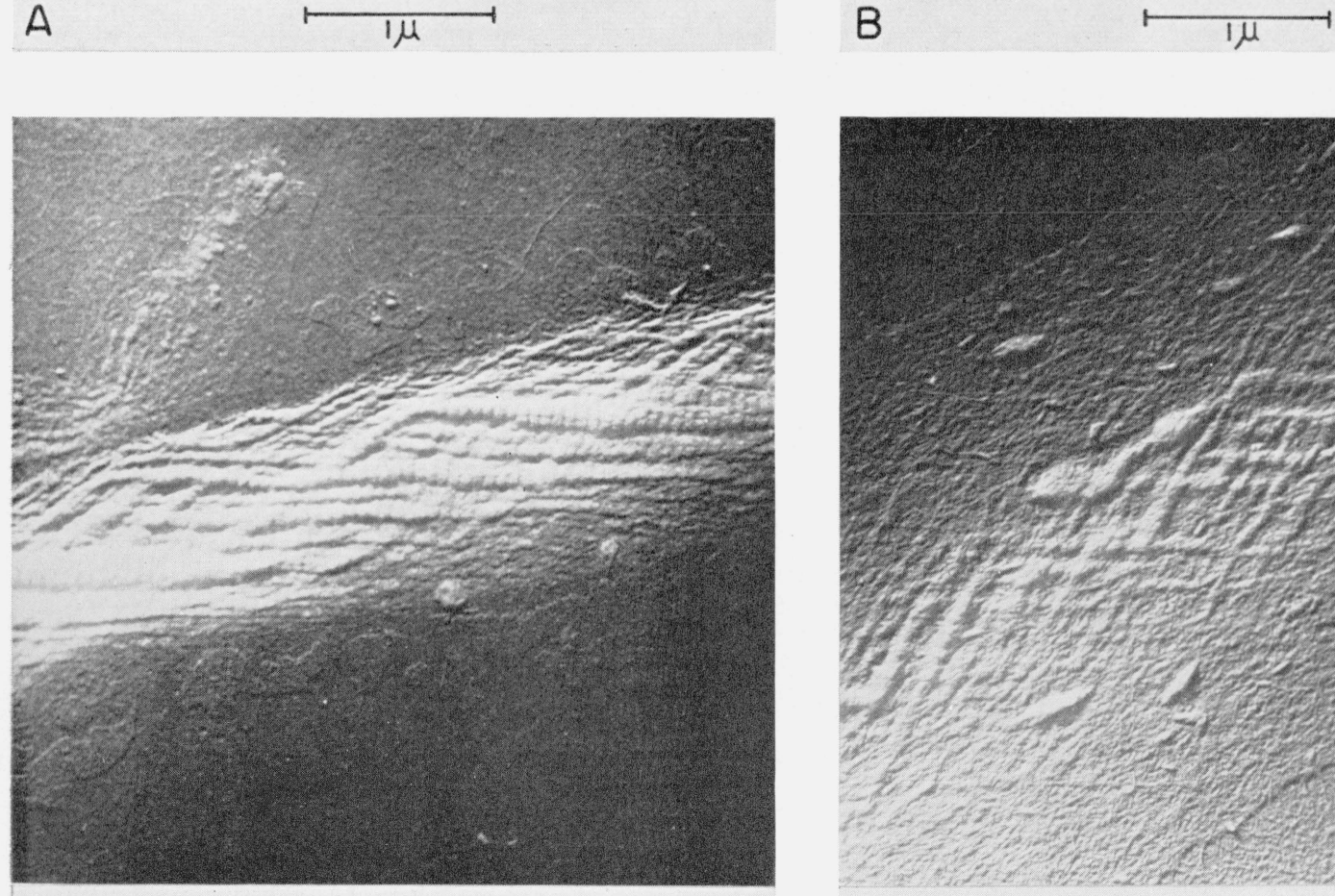

C
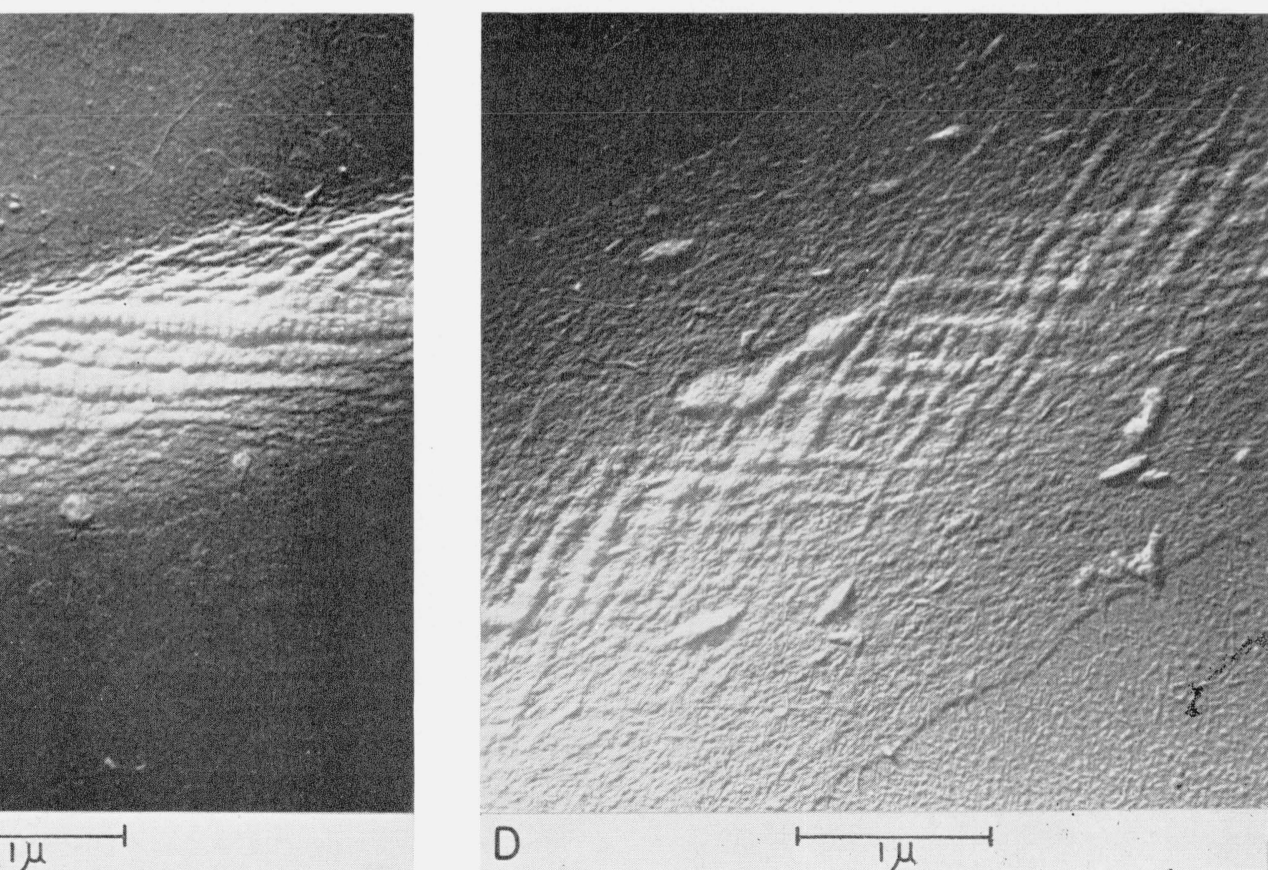

D

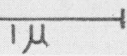

FiguRE 2. Electron micrographs of palladium-shadowed specimens of opened collagen fibrils of kangaroo iail tendon, dispersed in water.

A. Left-handed helical configuration in a single fibril.

. Single collagen fibril showing a cross-hatched appearance caused by the superposition of the upper and lower elements in the helical structure.
B. Right-handed helical configuration in three individual fibrils.

D. Single collagen fibril showing a more opened, flattened, and degraded structure resulting from additional treatment in a $1 \%$ acetic acid solution. 


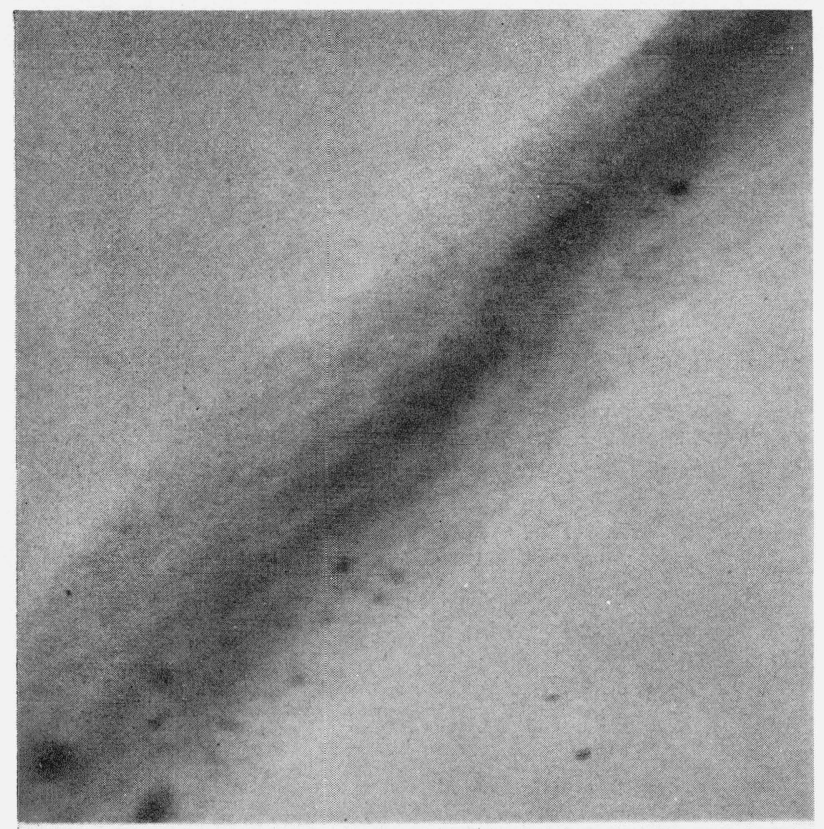

A

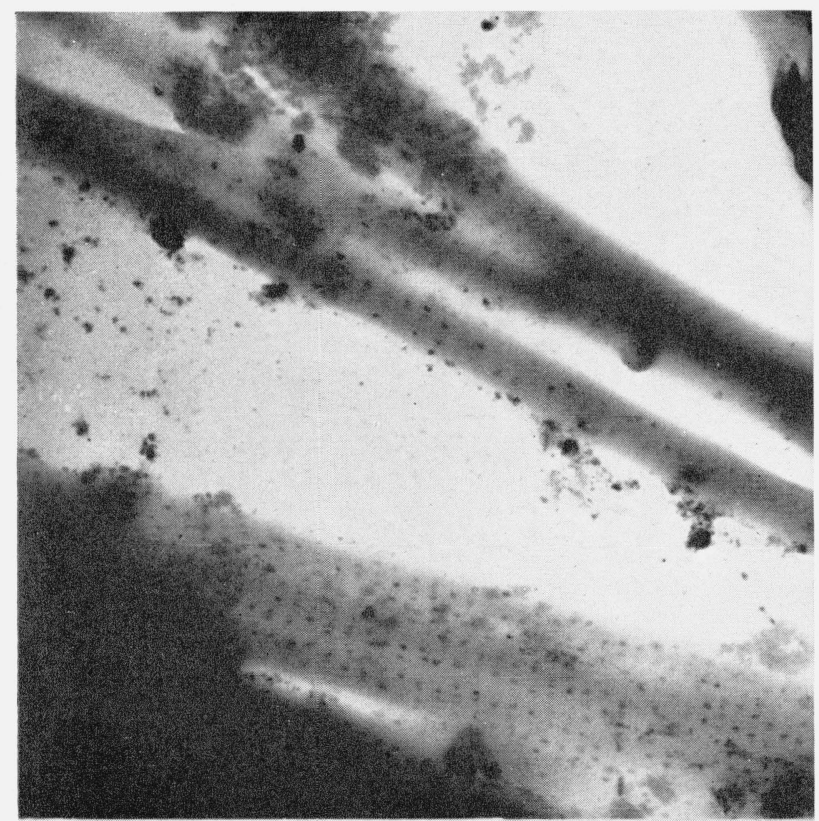

C

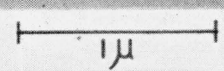

$\longmapsto$
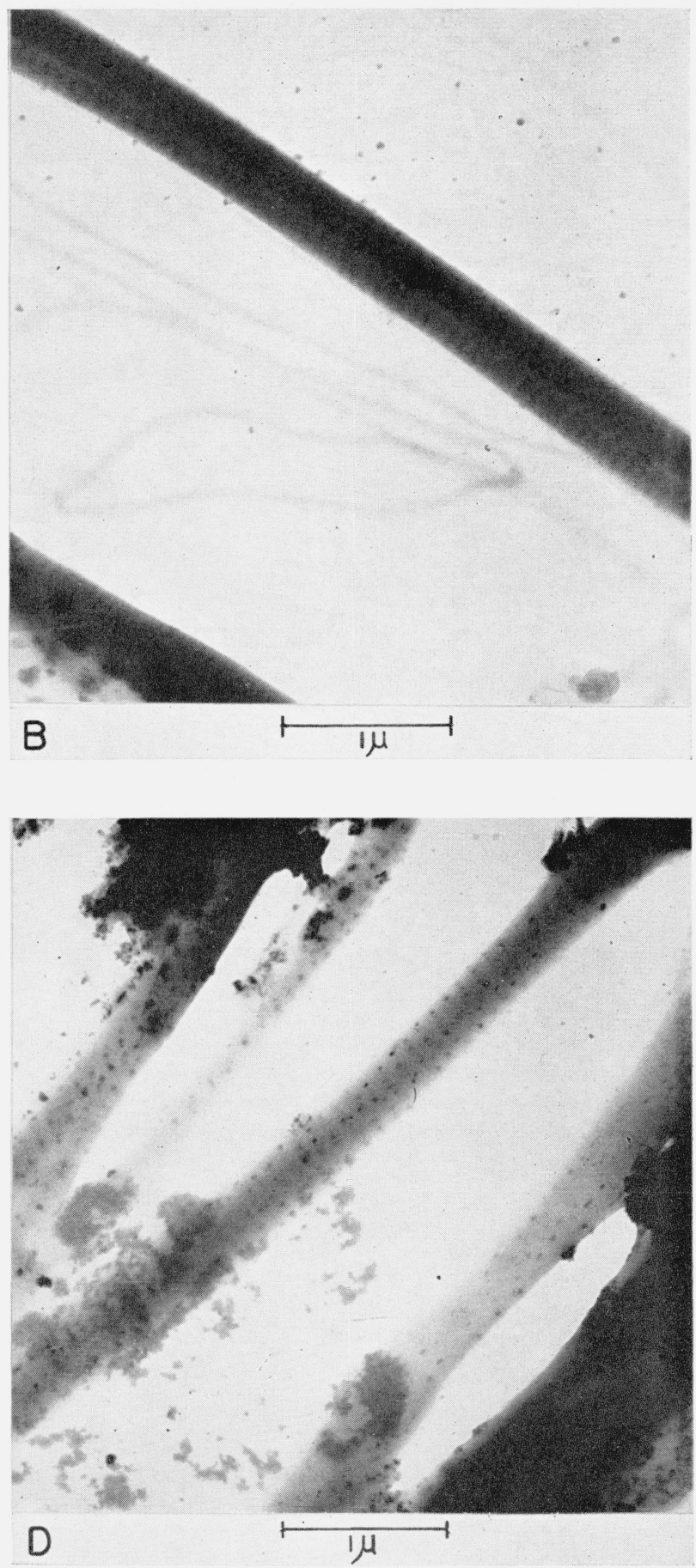

FIGURE 3. Electron micrographs of collagen fibrils of kangaroo tail tendon before and after exposure to liguid mercury under a pressure of 10,800 psi, dispersed in water.
A. Single untreated collagen fibril showing random particles of dirt in an opened structure. C. Collagen fibrils after pressure treatment showing oriented masses of mereury
occluded in the fibrillar structure.

B. Pair of collagen fibrils after exposure to mercury, showing evidence of structural preservation and preferential electron staining. Rows of opaque areas

D. Collagen fibrils after pressure treatment showing a regular arrangement of mercury masses. 


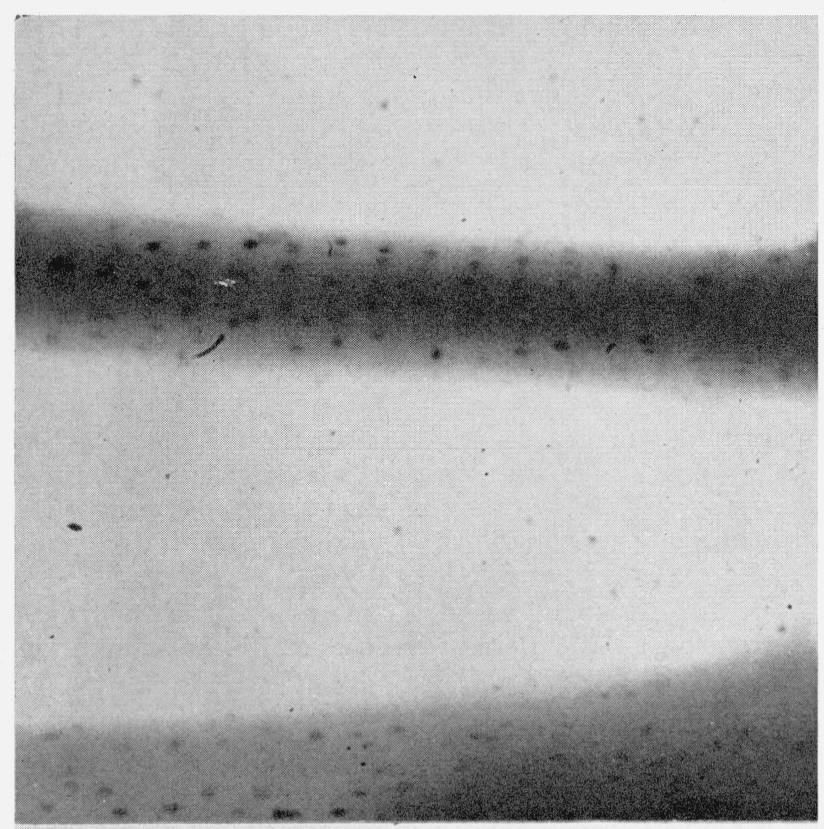

A
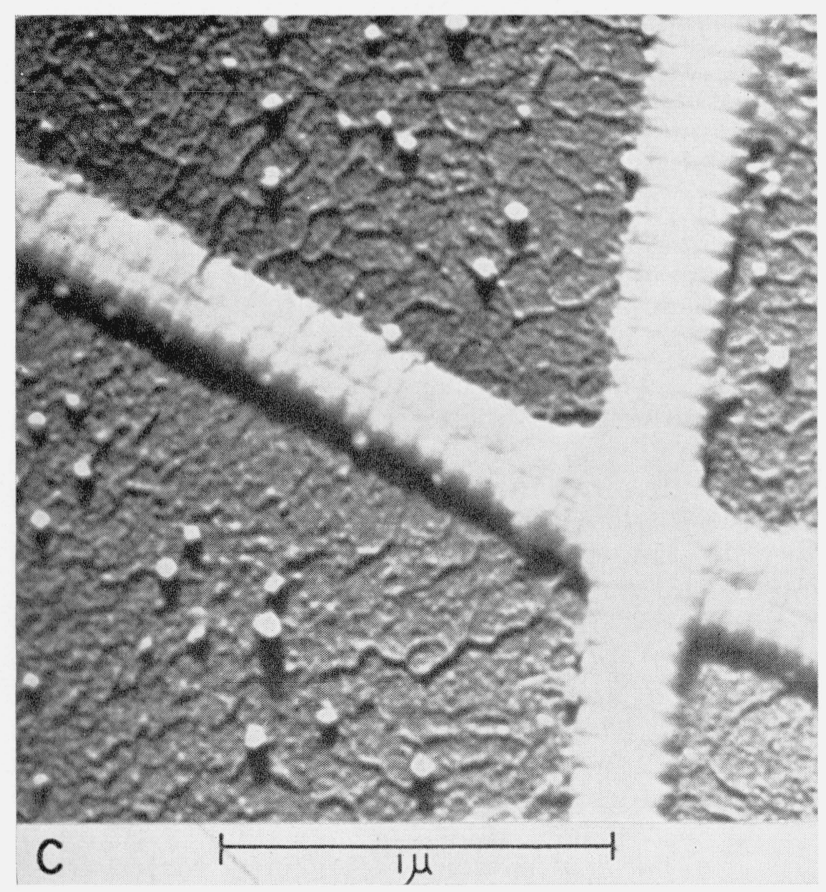
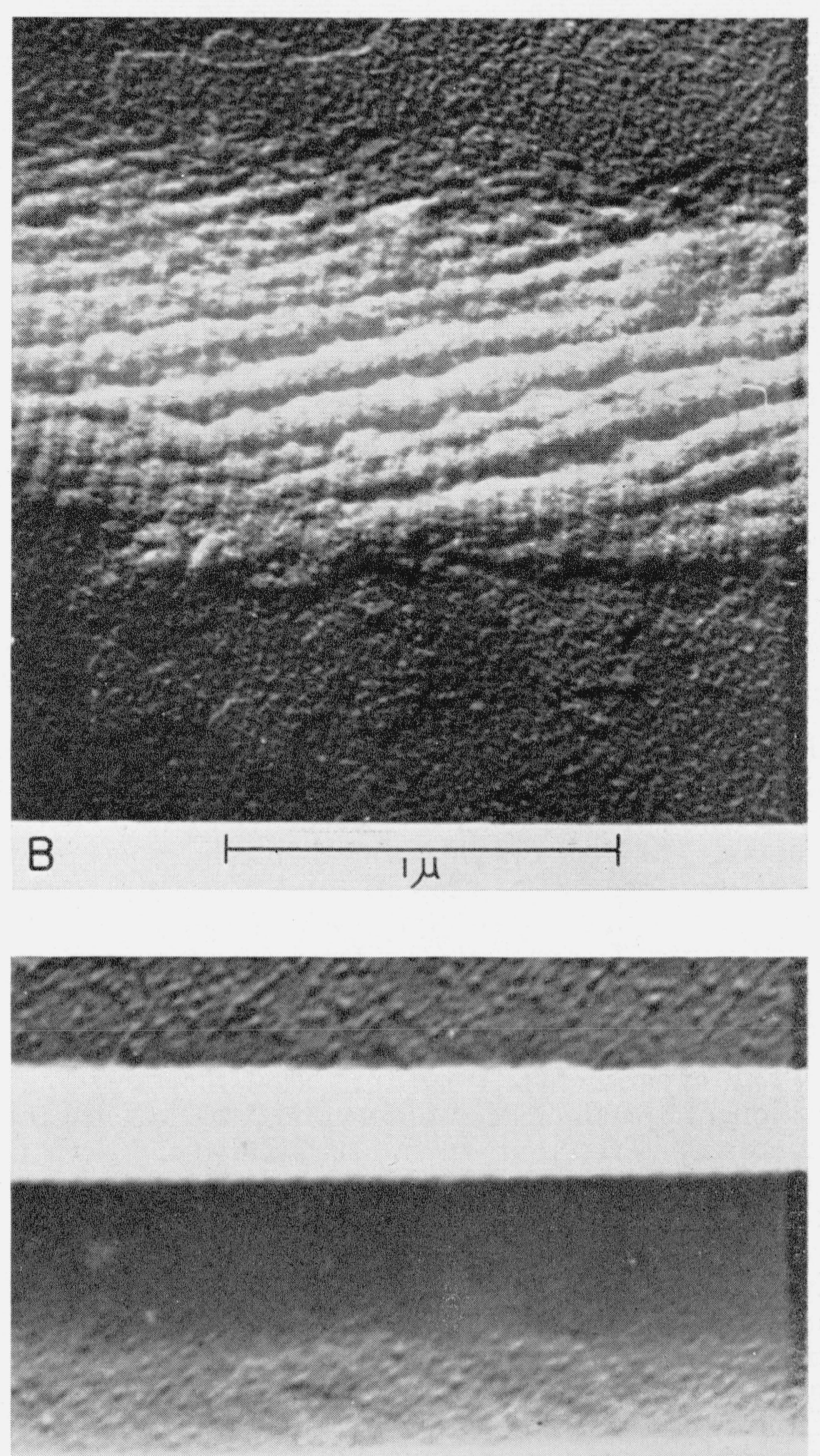

FIgURE 4. Electron micrographs of collagen from kangaroo tail tendon dispersed in water.

A. Single collagen fibril showing individual masses of mercury approximately $250 \mathrm{~A}$ in width and $350 \mathrm{~A}$ in length arranged in rows approximately $9^{\circ}$ from the perpendicular to the fibril axis and spsced about $1000 \mathrm{~A}$ apart. Another helical configuration of these opaque areas making an angle of about $18^{\circ}$ with respect to the fibril axis may also be discerned.

C. Palladium shadowed collagen fibrils after treatment with mercury under hydrostatic pressure of 10,800 psi. Colloida! masses in the background may be mercury released_from the impregnated collagen.
B. Single palladium shadowed collagen fibril showing a left-handed helical con. figuration. Elements of the helix make an angle of about $18^{\circ}$ with respect to the fibril axis. The opened, flattened structure demonstrates probable to the fioril
pore sites.

D. Two single palladium shadowed collagen fibrils after pressure impregnation with mercury showing a preserved three-dimensional structure and heavy electron staining. 
as 10,800 psi. Following this, the tendon was removed from the pressure vessel, cut into smaller pieces, and beaten in clean tap water in an electric food blender for $10 \mathrm{~min}$. The temperature of the water never exceeded $35^{\circ} \mathrm{C}$. Other specimens of the air-dried tendon were not subjected to the mercury treatment, but were dispersed in the blender in the above manner. In addition, some of these specimens were treated with acetic acid. Enough acid was added to the water in the blender to make a 1-percent solution, and the tendon was then given an additional minute of beating.

Drops of the suspension were pipetted onto collodion-covered specimen screens and dried in room air (conditioned at $22^{\circ} \mathrm{C}$ and 50-percent relative humidity). The specimens selected for shadowcasting were evacuated to a pressure of about $10^{-5} \mathrm{~mm}$ of $\mathrm{Hg}$ or less. A calculated thickness of $20 \mathrm{~A}$ of palladium was deposited in such a manner as to create shadows, the lengths of which were four times the altitude of the particle casting the shadows. The RCA type EMU-50kv electron microscope was used in this study. Electron optical magnifications of 10,000 diameters were used in recording the images. In the case of the shadowed specimens, negative prints were used in the figures illustrated, whereas in the case of the unshadowed specimens, positive prints were used.

\section{Results and Discussion}

The experimental procedure used for the preparation of specimens for observation in the electron microscope resulted in a suitable dispersion of collagen fibrils. In the case of the kangaroo tail tendon not subjected to mercury at elevated pressures, some of the fibrils showed evidence of having been laid open, while in the same preparation some of the fibrils showed no evidence of such disruption. Specimens treated with acetic acid always showed a considerable amount of extention and degradation. In the case of the mercury-impregnated material, no evidence of an opened structure was observed. The electron micrographs shown in figures 1 to 4 have been selected to show the presence of probable pore sites occurring within the fibril structure. Figure 1, A, represents a poorly dispersed group of collagen fibrils obtained from kangaroo tail tendon. Mercury probably would reach the pores existing between fibrils. These pores would most likely be of a larger order of magnitude than those existing within a single fibril. Figure $1, \mathrm{~B}$, shows shadowed parts of two individual fibrils that have the characteristic cross striations typical of collagen. Figure 1, C, shows two pairs of fibrils exhibiting alternate registry of the cross striations. Parallel registry also is commonly observed. Figure $1, \mathrm{D}$, shows the fraying that often occurs at the ends of fibrils.

The single fibrils in figure 2 appear to have been opened and somewhat disrupted. With the exception of figure 2, D, which was treated with acetic acid, the treatment was no different for these specimens than for those illustrated in figure 1. A left-handed or right-handed helical configuration seems to be indicated in these micrographs. The helix axis is the fibril axis, and the helix angle is approximately $72^{\circ}$. In other words, the helical elements, as viewed in the micrographs, make an angle of approximately $18^{\circ}$ with respect to the longitudinal axis of the fibril. Figure 2, A, exhibits a left-handed helical structure; 2 , B, shows three individual, opened fibrils with a right-handed helical structure. The crosshatching in figures $2, \mathrm{C}$, and $2, \mathrm{D}$, is indicative of an opened and collapsed helical structure in which the upper and lower elements may be seen. No special significance can be given to helix direction, inasmuch as approximately an equal number of occurrences of left-handedness and right-handedness was observed. The electron micrographs published by other workers concerned with the microstructure of collagen, for example [4, p. $235 ; 12 ; 13$, p. 204; 14 ; 15 , p. 107], also indicate a formation that could be interpreted as an opened, flattened, helical structure.

The possibility that the helices observed are artifacts resulting from the method of dispersing the individual fibrils appears to be unlikely, as no single preferred helical direction was produced by the blender. As was pointed out in section 1, on the basis of X-ray evidence, helical configurations have been proposed for the structure of the collagen molecule. Acknowledging the possibility that the helical configuration may result from the drying-out process in the method of mounting the specimen, and realizing that the helical structures described for the molecule are at least an order of magnitude smaller than those observed in the electron micrographs, it is still possible that the inherent configuration of the molecule is reflected in the larger subfibrillar structures observed in these micrographs and that these helical formations are real.

The disrupted, opened structure of fibrils seen in figure 2 , nevertheless, suggests evidence that pores exist within the fibril structure. The distorted structures observed in these opened fibrils do not permit a delineation of the shape and size of these pores, but demonstrate the existence of probable pore sites in the undistorted structure. Examination of the mercury-impregnated material provides additional information about the location, size, and arrangement of these pores (fig. 3). Figure 3, A, illustrates a single, unshadowed collagen fibril that has had no contact with mercury. The incidental particles of debris associated with the preparation have a random configuration. When kangaroo tail tendon was placed in water containing a drop of mercury and dispersed in the blender for $10 \mathrm{~min}$., none of the collagen fibrils showed evidence of mercury impregnation. The mercury appeared as small droplets in the background, and any association with the collagen was random. The pair of fibrils shown in figure $3, \mathrm{~B}$, have been subjected to mercury under hydrostatic pressure and show the fibrils to be partially stained and structurally preserved. There is no apparent opening or disruption. of the fibrils. There is, moreover, a regular lodgment of electron optically opaque material, presumably mercury or a compound of mercury. 
These denser spots are arranged in a more or less periodic fashion. Figures 3, C, and 3, D, show similarly treated kangaroo tail tendon, where the individual fibrils do not show the same degree of staining as those in figure 3, B. The spots of mercury, however, are seen in better contrast and exhibit a distribution similar to that observed in figure $3, \mathrm{~B}$.

An enlarged view of a section of figure $3, \mathrm{D}$, is shown in figure $4, \mathrm{~A}$. Although no direct comparisons can be made, examination of the helical fibrillar structure seen in figure 4 , B, shows many probable pore sites across and along the helix. The number of spots in a given row across the fibril seen in figure $4, \mathrm{~A}$, is considerably less. This may be accounted for in two ways. Many of the pore sites seen in figure 4, B, are probably considerably smaller in radius than $100 \mathrm{~A}$ in the unopened state. Within the limits of the apparatus and procedure used in the porosimeter method [11], the maximum pressures that could be obtained would theoretically penetrate cylindrical pores down to only $100 \mathrm{~A}$ in radius. It is also expected that some of the mercury might be dislodged when the pressure was released and also in the subsequent treatment during specimen preparation. If parallel lines were ruled across the fibril axis connecting these spots, in figure $4, \mathrm{~A}$, one set would be about $1000 \mathrm{~A}$ apart, with a helix angle of about $9^{\circ}$. The characteristic cross striations observed in collagen, figure $1, \mathrm{~B}$, also show this same helix angle. Another set of lines following a helical path about the fibril axis would be about $750 \mathrm{~A}$ apart and make a helix angle of about $72^{\circ}$. This is the same value obtained for the helix angle in the case of the opened, disrupted fibrils not impregnated with mercury. The individual spots are of the order of $250 \mathrm{~A}$ in width and $350 \mathrm{~A}$ in length, the longer dimension being parallel to the fibril axis. For a comparison with the circular cross section of the pore assumed for calculating the distribution curve for kangaroo tail tendon (11), these dimensions would average out to a radius of the order of $150 \mathrm{~A}$.

Analysis of the pore-size distributions in kangaroo tail tendon, as well as in the finished sole leather, splits of crust leather, rawhide, and sharkskin showed that a significant volume and number of pores were concentrated in the small pore-size range, 100 to $1000 \mathrm{~A}$, in radius. It would appear then that these micrographs provide a visual experimental confirmation of the existence and probable size of pores in collagen fibrils, deduced from theoretical considerations of distributions obtained from porosimeter measurements on collagen and leather [11]. Additional information about the shape, location, and arrangement of such pores is also provided.

Observation of the location of the denser spots in figure $4, \mathrm{~A}$, indicates that some of the mercury is lodged in the peripheral surface of the fibril. The fact that some spots appear more opaque than others may be a result of the additional scattering caused by the fibril in the transmission of the electron beam. The darker spots represent areas where the electron beam had already penetrated the fibril and received little additional scattering from the collagen after encountering a discrete mass of mercury.

The variations in opaqueness to the electron beam also may be a result of variations in the thicknesses of the masses of mercury. From the electron micrographs, no conclusive evidence may be obtained as to how far the mercury has penetrated within the fibril. Information obtained from shadow-cast mercury-impregnated fibrils, figure 4, C, indicates that the masses did not protrude to any observable extent.

No ideal single procedure for fixing and staining has been found that provides optimum preservation of all features found in biological material. The fixatives and stains that have been so universally accepted for use in light microscopy are not, in general, satisfactory for use in electron microscopy. Contrast in light microscopy depends upon the selective absorption and transmission of chemically stained histological features. Electron microscopy develops image contrast that depends largely upon differential electron scattering produced by variations in the thickness and density of the object. Among the important variables employed in the accepted procedures for staining and fixing are chemical composition, concentration, $\mathrm{pH}$, exposure time, and to a lesser extent, variation of temperature. For example, buffered osmium solutions or vapor and aqueous solutions of formaldehyde have been used with considerable success in the electron microscopy of biological material.

From the evidence observed in the unshadowed and shadowed collagen fibrils of kangaroo tail tendon exposed to mercury at a pressure of $10,800 \mathrm{psi}$, it appears that a considerable degree of staining without undue distortion of the characteristic structure of collagen has been attained. The shadow lengths in figure $4, \mathrm{D}$, demonstrate that the three-dimensional structure of the collagen fibril apparently has been preserved. The opaqueness of the individual collagen fibrils to the electron beam is also apparent in figures $4, \mathrm{~A}$, and 4, D. This suggests that hydrostatic pressure may possibly be another important variable in the processes of staining and fixing materials for electron microscopy. It offers a potential advantage in producing physical embedment of liquid substances, apparently without undue structural distortion.

The authors sincerely appreciate the helpful suggestions of Jerome Gross of the Harvard Medical School and Massachusetts General Hospital, Francis H. C. Crick of the Polytechnic Institute of Brooklyn, Albert J. Dalton of the National Cancer Institute, and Charles E. Weir of the National Bureau of Standards. 


\section{References}

[1] R. Borasky, Guide to the literature on collagen, AIC-278 (U. S. Department of Agriculture, Eastern Regional Research Laboratory, Philadelphia, Pa., July 1950).

[2] M. Swerdlow, Electron microscopy (review), Anal. Chem. 26, 34 (1954).

[3] R. S. Bear, The structure of collagen fibrils, Advances in Protein Chem. VII, 69 (1952).

[4] J. T. Randall (Editor), Nature and structure of collagen (Academic Press, Inc., New York, N. Y., 1953).

[5] J. H. Highberger, A half century of progress in collagen chemistry, J. Am. Leather Chemists' Assoc. 48, 704 (1953)

[5a] W. Grassman, Electron optical and chemical studies on the structure of collagen, p. 195, The chemical structure of proteins, (a Ciba Foundation symposium, J. and A. Churchill, Ltd., London, England, 1953).

[6] L. Pauling and R. B. Corey, The structure of fibrous proteins of the collagen-gelatin group, Proc. Nat. Acad. Sci. 37, 272 (1951); see also, A discussion on the structure of proteins, Proc. Roy. Soc. (London B) 141, p. 1 (1953)

[7] C. Cohen and R. S. Bear, Helical polypeptide chain configuration in collagen, J. Am. Chem. Soc. 75, 2783 (1953).
[8] F. H. C. Crick, A structure for collagen, J. Chem Phys. 22, 347 (1954)

[9] F. O. Schmitt, C. E. Hall, and M. A. Jakus, Electron microscope investigations of the structure of collagen, J. Cellular Comp. Physiol. 20, 11 (1942)

[10] R. Oehler, S. Dahl, and T. J. Kilduff, Treatment of leather with polyisobutylene, J. Am. Leather Chemists' Assoc. 4\%, 642 (1952).

[11] R. R. Stromberg, Pore-size distribution in collagen and leather by the porosimeter method, J. Research NBS 54, 73 (1955) RP2567.

[12] F. O. Schmitt and J. Gross, Further progress in the electron microscopy of collagen, $\boldsymbol{J}$. Am. Leather Chemists' Assoc. 43, 658 (1948).

[13] R. W. G. Wyckoff, Electron microscopy technique and applications, p. 203 (Interscience Publishers, Inc., New York, N. Y., 1949)

[14] C. E. Hall, Introduction to electron microscopy, p. 403 (McGraw-Hill Book Co., Inc., New York, N. Y., 1953).

[15] G. C. Nutting and R. Borasky, Electron microscopy of collagen, J. Am. Leather Chemists' Assoc. 43, 96 (1948).

Washington, August 6, 1954. 